\title{
Psicocirurgia para tratamento de transtorno obsessivo-compulsivo
}

\author{
Leila Maria Da Róz', Eduardo Santamaria Carvalhal Ribas', Gerson \\ Ballester², Eberval Gadelha Figueiredo², Manoel Jacobsen Teixeira²
}

\section{RESUMO}

O transtorno obsessivo-compulsivo (TOC) é uma patologia incapacitante tanto na esfera social como na ocupacional. Está associado a uma morbidade bastante alta quando comparado a outras condições psiquiátricas e estima-se atualmente uma prevalência de $2 \%$ a $3 \%$ na população. Alguns estudos sugerem uma provável relação com uma disfunção do córtex frontal e do cíngulo, envolvendo o circuito de Papez. Esse circuito parece mediar os sintomas ansiosos, visto que lesões cirúrgicas dessas áreas afetam a ansiedade geralmente associada ao TOC. A neurocirurgia ablativa para o tratamento de distúrbios psiquiátricos foi muito utilizada até o desenvolvimento de psicofármacos efetivos. Recentemente, com os avanços técnicos do procedimento cirúrgico, auxiliado pelo conhecimento neurofisiológico, a psicocirurgia tornou-se mais precisa e segura. Porém, sua indicação se restringe como uma alternativa ao tratamento de pacientes com TOC refratário à terapia comportamental el ou medicamentosa.

\section{PALAVRAS-CHAVE}

Transtorno obsessivo-compulsivo/classificação, transtorno obsessivo-compulsivo/etiologia, transtorno obsessivo-compulsivo/fisiopatologia, psicocirurgia, procedimentos cirúrgicos minimamente invasivos, procedimentos neurocirúrgicos.

\section{ABSTRACT}

Psychosurgery for the treatment of obsessive-compulsive disorder

The obsessive-compulsive disorder $(O C D)$ is a both socially and occupationally incapacitating disease. It is associated with a quite high morbidity if compared to other psychiatric conditions and currently it is estimated a lifetime prevalence rate of $2 \%$ to $3 \%$. Some studies suggest a probable relation with a dysfunction in the cingulate and the frontal cortex, involving the Papez circuit. Anxious symptoms seem to be mediated by that circuit, considering surgical lesions of these areas affect the anxiety usually associated with OCD. The ablative neurosurgery for the treatment of psychiatric disorders was widely used until the development of effective psychopharmacological medication. Recently, thanks to the technical advances of the surgical procedure, aided by the neurophysiological knowledge, the psychosurgery became more accurate and safer. Yet, its indication is restricted as an alternative treatment to refractory $O C D$ patients.

\section{KEYWORDS}

Obsessive-compulsive disorder/classification, obsessive-compulsive disorder/etiology, obsessivecompulsive disorder/physiopathology, psychosurgery, surgical procedures, minimally invasive, neurosurgical procedures.

\section{Introdução}

O transtorno obsessivo-compulsivo (TOC) interfere significativamente nas atividades domésticas, sociais e profissionais cotidianas dos pacientes. Atualmente, recebe especial atenção da comunidade psiquiátrica, pois revela-se menos raro do que se pensava até recentemente. ${ }^{24} \mathrm{~A}$ ineficácia do tratamento convencional, em alguns casos, leva à procura de outros métodos, sendo a psicocirurgia uma das alternativas.

Quadros obsessivo-compulsivos têm sido descritos há pelo menos 300 anos, sendo muitas vezes associados à insanidade ou à religião, porém a hipótese de que estariam vinculados a uma disfunção cerebral foi proposta apenas na última metade do século XIX. ${ }^{5}$

As primeiras teorias envolviam a ideia de possessão por forças estranhas e a necessidade da realização de rituais de exorcismo por médicos-bruxos, místicos ou líderes religiosos visando à expulsão dos invasores indesejados. Freud desenvolveu a primeira hipótese racional, conceituando a sintomatologia do TOC por meio de questões psicossexuais relacionadas à teoria da libido. Alguns teóricos enfatizaram elementos particulares, como a raiva reprimida, mas a ideia de controle de impulsos sexuais 
hostis e inaceitáveis ainda predominava. Mesmo antes de Freud, o comportamento bizarro do indivíduo obsessivo-compulsivo já fora relacionado à sua aflição, mas só recentemente o valor adaptativo de tal comportamento foi reconhecido mais claramente: as preocupações insistentes do indivíduo ocupam de tal maneira sua atenção, que ele não consegue dedicar-se a qualquer outro tipo de pensamento, distraindo-se de todo o restante. ${ }^{32}$

\section{Epidemiologia}

Apesar de o conceito de TOC estar estabelecido há muito tempo, os critérios diagnósticos utilizados nas investigações são relativamente recentes. Isso se deve parcialmente à possibilidade de seus sintomas ocorrerem concomitantemente aos de outras doenças e, portanto, ser discutível até que ponto constituem fenômenos distintos. ${ }^{4,24}$ Além disso, há grande dificuldade no estabelecimento de métodos diagnósticos para o cálculo da prevalência dessa patologia, já que o modo de avaliação dos indivíduos é muito subjetivo. ${ }^{9}$

Os primeiros estudos clínicos relatavam prevalências próximas de $0,3 \%$ e $1 \%{ }^{9}$, porém, atualmente, estudos epidemiológicos, baseados no DIS (Diagnostic Interview Schedule) estimam uma prevalência ao longo da vida entre $2 \%$ e $3 \%{ }^{23,30,33}$ e uma prevalência em seis meses entre $1 \%$ e $2 \%$ na população adulta de diversos países, entre eles os Estados Unidos. ${ }^{4,12,24}$

No Brasil, as prevalências estimadas para homens e mulheres são respectivamente de $1,7 \%$ e $0,7 \%$ em Porto Alegre e de 0,5\% e 0,4\% em Brasília. ${ }^{9}$ Apesar de diversos estudos utilizando o DIS demonstrarem uma maior proporção de indivíduos do sexo feminino, ${ }^{4,24}$ há indícios de que estes não possuem maior predisposição para o TOC; na verdade, percebe-se que geralmente os homens apresentam o início dos sintomas mais precocemente. De modo geral, a doença afeta pessoas jovens: $60 \%$ dessas reportam os primeiros sintomas antes dos 25 anos e 30\% delas apresentam-nos entre os 5 e 15 anos..$^{9,29}$

\section{Quadro clínico}

Os portadores de TOC podem apresentar obsessões, compulsões ou ambos os sintomas conjuntamente. As obsessões são definidas pelo DSM-IV como a presença intrusiva de pensamentos, impulsos ou imagens recorrentes e persistentes, frequentemente repugnantes, levando à ansiedade ou ao sofrimento do paciente. Essa intrusão deve ser maior que a experimentada em preocupações habituais. Além disso, o paciente deve reconhecer os pensamentos ou impulsos obsessivos como produtos de sua própria mente e esforçar-se para ignorá-los ou suprimi-los, ou neutralizá-los por meio de outro pensamento ou ação. Já as compulsões são caracterizadas por comportamentos repetitivos ou atos mentais executados pelo paciente em resposta a uma obsessão ou conforme regras que devem ser rigidamente empregadas. ${ }^{4}$ Elas têm o objetivo de prevenir ou reduzir o sofrimento e/ou ansiedade causados pelas obsessões ou algum evento ou situação que cause temor ${ }^{1,432}$, entretanto devem ser claramente exageradas ou incoerentes com o que se deseja neutralizar ou evitar, e isso deve ser reconhecido pelo paciente no decorrer do transtorno. Ainda segundo o DSM-IV, para a caracterização do TOC, as obsessões ou compulsões devem ser marcadamente perturbadoras, consumir mais de uma hora por dia e interferir nas atividades normais do paciente. ${ }^{4}$

\section{Qualidade de vida}

Apesar da existência de vários estudos epidemiológicos sobre a morbidade relacionada ao TOC e de insistentes pesquisas na origem dos sintomas e dos transtornos funcionais causados por ele, poucos realmente se empenharam na avaliação de seu impacto na qualidade de vida de seus portadores. Em um desses, pacientes com TOC de moderado a intenso, não medicados, foram comparados à população em geral dos Estados Unidos e a pacientes com depressão ou diabetes. Os portadores de TOC obtiveram uma pontuação média mais alta em todos os domínios relacionados à saúde física como determinante da qualidade de vida (função física, limitação devido a problemas físicos e dores no corpo) do que pacientes com diabetes e depressão, porém semelhante à da população em geral. Em contraste, em todos os domínios de saúde mental (função social, limitação devido a problemas emocionais, e saúde mental), os pacientes com TOC obtiveram uma pontuação média bem abaixo da população em geral (quanto mais grave o transtorno, mais baixa a pontuação), já a pontuação média dos com diabetes e depressão foi semelhante. Assim, a intensidade do TOC foi negativamente relacionada à função social, demonstrando um importante impacto negativo dessa moléstia na qualidade de vida de seus portadores. ${ }^{23} \mathrm{Um}$ agravante desse problema é que geralmente há um grande período entre os primeiros sintomas e o início do tratamento apropriado. ${ }^{24}$ Além disso, pode haver refratariedade ao tratamento convencional, casos em que a neurocirurgia surge como uma alternativa, já que atualmente se mostra mais precisa e segura no tratamento de pacientes psiquiátricos. 


\section{Fisiopatologia}

A causa do TOC ainda não foi esclarecida, embora não pareça ser determinado por nenhum fator etiológico específico. Possivelmente, resulta da interação de múltiplos fatores: genéticos, biológicos e sistemas familiares. ${ }^{29}$ Contudo, a literatura neurocirúrgica sugere provável associação dessa perturbação a uma disfunção do córtex frontal e cíngulo, crendo-se no envolvimento do circuito de Papez, do qual essas estruturas fazem parte, na mediação de sintomas característicos de ansiedade e de outros distúrbios emocionais, conforme descrito.

Algumas manifestações obsessivo-compulsivas estão começando a ser definidas por meio de sua fisiopatologia, no entanto podem representar apenas alterações intermediárias, e não a etiologia primária determinante. Há fortes evidências da associação de fatores neurobiológicos e psicológicos na patogênese do TOC..$^{12,24}$ Relatos de distúrbios neurológicos em que há comprometimento do lobo frontal, núcleo caudado, globo pálido, e possivelmente do lobo temporal, associados a sintomas de TOC, indicam o possível envolvimento de tais estruturas na gênese dos sintomas. ${ }^{33}$

Os circuitos possivelmente relacionados à patogênese de transtornos afetivos, ansiosos e psicóticos, em sua maioria, envolvem conexões entre o giro do cíngulo e o neocórtex do lobo frontal. ${ }^{34}$ Os transtornos obsessivo-compulsivos, por apresentarem sintomas variados, possuem diversas hipóteses descritas, envolvendo estruturas anatômicas distintas.

Para melhor compreensão da base anatômica do TOC, é necessário citar os três principais circuitos límbicos: o circuito medial, o basolateral e o de reação de fuga e luta. Dentre esses, somente os dois primeiros parecem estar envolvidos em transtornos afetivos e ansiosos, sendo o circuito límbico medial o clássico circuito de Papez, com conexões do giro do cíngulo ao hipocampo, fórnix, corpos mamilares e núcleo anterior do tálamo e então ao córtex orbitofrontal e núcleo septal. $\mathrm{O}$ circuito basolateral envolve o lobo temporal anterior, amígdala, núcleo dorsomedial do tálamo e córtex orbitofrontal. ${ }^{34} \mathrm{O}$ circuito de Papez parece mediar os sintomas ansioso $\mathrm{s}^{15,33} \mathrm{e}$ o basolateral, o comportamento obsessivo-compulsivo, principalmente porque lesões cirúrgicas que alteram as relações funcionais dessas áreas parecem melhorá-los. ${ }^{33,34} \mathrm{~A}$ utilidade clínica desses procedimentos parece ser baseada mais na análise pragmática de sua eficácia em relatos anteriores do que um completo conhecimento de sua base científica. ${ }^{34}$ Um dado concreto é que a estimulação elétrica do giro do cíngulo em pacientes com epilepsia refratária à medicação, com eletrodos implantados a fim de localizar o foco de origem da crise, induz movimentos motores repetitivos e estereotipados similares aos de rituais compulsivos. $^{33}$
Uma teoria que propõe o envolvimento de uma alça fronto-estriato-pálido-talâmico-frontal afirma que o TOC poderia ser o resultado tanto de desequilíbrios da atividade modulatória da conexão fronto-caudado-pálido-talâmica como de uma alteração da atividade primária da conexão órbito-frontal-talâmica. ${ }^{33}$ Lesões na área órbito-frontal, no trato órbito-frontal-talâmico ou nos núcleos talâmicos anteriores mediais melhoram os sintomas obsessivo-compulsivos, apresentando o provável papel desse circuito na patogênese do TOC.

A anatomia cerebral encontra-se, muitas vezes, alterada em pacientes com TOC, como demonstrado em estudos de imagem com tomografia computadorizada (TC) e ressonância magnética (RM) de alta resolução, com redução do volume médio do cíngulo, comparado a controles normais. Entretanto, não há descrições de alteração estrutural evidente da substância branca da área frontal em alguns pacientes. ${ }^{33}$

$\mathrm{O}$ achado mais consistente acerca da fisiopatologia do TOC aponta para uma anormalidade na neurotransmissão mediada por serotonina, ${ }^{12,24}$ porque há boa resposta terapêutica com o uso de inibidores seletivos da recaptação de serotonina. O uso de inibidores da recaptação de noracrenalina ou de antagonistas dopaminérgicos não demonstra melhora do quadro. ${ }^{12,24}$ Estudos com tomografia por emissão de pósitrons (PET) demonstraram a ocorrência de mudanças neurobiológicas no sistema nervoso central tanto durante tratamento farmacológico como durante psicoterapia. ${ }^{12,24}$ Já níveis anormais de neurotransmissores e neuromoduladores foram encontrados no fluido cerebroespinhal de pacientes com TOC não tratados, no entanto após tratamento efetivo ocorreu a normalização daqueles. ${ }^{24}$

A avaliação neuropsicológica frequentemente identifica anormalidades visuoespaciais. ${ }^{24} \mathrm{Um}$ estudo encontrou significativamente mais sinais de disfunção do sistema nervoso central (por exemplo: anormalidades na coordenação motora fina e na função visuoespacial e movimentos involuntários) em pacientes com TOC em relação a controles. Esse mesmo estudo reportou uma correlação entre sinais mais brandos e a gravidade das obsessões, bem como um excesso de anormalidades localizadas à esquerda e déficit visuoespacial, ambos consistentes com disfunções do hemisfério cerebral direito. Também existem relatos de maior déficit de memória não verbal recente, talvez relacionado à hipótese preexistente da maior associação da área mesial temporal direita a memórias não verbais, sendo o hipocampo e a amígdala geralmente considerados como centralmente envolvidos na memória recente. O prejuízo da memória também foi identificado em humanos após lesões diencefálicas, do prosencéfalo basal e do giro do cíngulo. Portanto, há forte evidência de que os achados neuropsicológicos e os sintomas do TOC devem-se particularmente a disfunções dos lobos 
frontal e temporal e primariamente, mas não exclusivamente, do lobo temporal direito. ${ }^{33}$

\section{Indicação cirúrgica}

Tratamentos específicos como terapias comportamentais (resposta preventiva e a exposição in vivo) provaram ter sucesso em diminuir rituais compulsivos em $70 \%$ a $80 \%$ dos pacientes. O uso de medicação deve ser visto como uma alternativa em casos irresponsivos à primeira forma de tratamento, contudo, se o TOC ainda se apresentar refratário, a psicocirurgia torna-se uma opção de tratamento. Assim, pacientes extremamente doentes e incapacitados são encaminhados para o procedimento neurocirúrgico após serem selecionados por rigoroso critério. ${ }^{22,26}$

A neurocirurgia psiquiátrica ("psicocirurgia") é definida como uma forma de neurocirurgia funcional destinada ao tratamento de distúrbios psiquiátricos, ocupando uma posição central, apesar de controversa, principalmente na década de 1940 e início dos anos 1950, especialmente nos Estados Unidos. No entanto, muitos desses procedimentos provocavam efeitos colaterais importantes, como hemorragia, epilepsia e a "síndrome do lobo frontal", caracterizada por apatia, impulsividade etc. ${ }^{20}$ Assim, em 1954, com a introdução da clorpromazina, um antipsicótico, houve uma conscientização a respeito das morbidades relacionadas à psicocirurgia. Já na década de 1960, esses procedimentos despertaram grande interesse, acompanhados por uma evolução contínua dos princípios estereotáxicos, refinamento da técnica e redução dos efeitos indesejáveis causados anteriormente. Próximo à década de 1970, novamente houve uma mobilização, devido a questões políticas, contra essa abordagem para tratamento de transtornos psiquiátricos. ${ }^{6,17}$

Recentemente, observa-se um novo interesse pelo tratamento cirúrgico de pacientes psiquiátricos, visto que os procedimentos neurocirúrgicos atuais são considerados relativamente seguros, com letalidade extremamente baixa e morbidade pós-operatória transitória, ${ }^{6}$ porém a indicação de psicocirurgia restringe-se àqueles em que o tratamento convencional não demonstrou eficácia. ${ }^{27,29}$

Os procedimentos cirúrgicos podem tanto atuar diretamente no circuito de Papez (cingulotomia anterior), em conexões entre o córtex frontal e o sistema límbico (tractotomia do subcaudado e capsulotomia), ou em ambos (leucotomia límbica). ${ }^{6}$

Atualmente, há quatro técnicas mais frequentemente utilizadas no tratamento de transtornos psiquiátricos, sendo cada uma associada a determinado centro, muitas vezes utilizando metodologias distintas para a seleção de pacientes, o que torna difícil a comparação entre os diversos resultados. ${ }^{29}$

Antes do abandono das formas mais conservadoras de tratamento, é prudente rever o diagnóstico, excluir qualquer outra morbidade psiquiátrica (dependência de droga, psicose ou transtorno de humor) e afastar os estressores psicossociais. Além disso, antes da indicação do procedimento cirúrgico, devem-se balancear os riscos da não intervenção (complicações sociais, físicas e psicológicas, incluindo risco de suicídio) e os da intervenção (disfunção do lobo frontal e complicações psicológicas, tais como alteração da personalidade, abuso de substâncias e suicídio), minimizados com as técnicas atuais. As indicações para a realização desses procedimentos são essencialmente as mesmas para as várias técnicas existentes e entre os diversos serviços médicos. $^{34}$

Abaixo se encontram relacionados os critérios de seleção em psicocirurgias para tratamento de TOC.

- Os sintomas preenchem os critérios para TOC.

- A doença persiste por mais de cinco anos.

- O paciente encontra-se na faixa etária entre 18 e 65 anos (em alguns serviços, realiza-se o procedimento em pacientes com 20 a 69 anos).

- O TOC causa importante sofrimento e tem considerável impacto psicossocial na vida do paciente.

- Os seguintes tratamentos falharam ou não foram tolerados: testes adequados de todos os inibidores da recaptação de serotonina (alguns centros exigem testes de apenas três) e de um inibidor da MAO; combinação de no mínimo um inibidor da recaptação de serotonina com dois outros agentes (lítio, clonazepam ou buspirona, um mês cada) e, em pacientes com tiques associados, um neuroléptico em dose baixa; no mínimo 20 horas de terapia comportamental.

- O paciente não tem um bom prognóstico sem a cirurgia.

- O paciente deve estar informado sobre o procedimento e consenti-lo, concordando com a realização de uma avaliação pré-operatória e de uma reabilitação pós-operatória.

- O médico que indicou a cirurgia dispõe-se a realizar o acompanhamento pós-operatório e a longo prazo.

É importante manter ou recomeçar os tratamentos farmacológicos e/ou psicológicos no pós-operatório, pois os pacientes podem apresentar-se responsivos a intervenções anteriormente sem sucesso. ${ }^{16,19}$

O paciente também deve apresentar condições físicas favoráveis; não apresentar história pregressa de convulsões, derrames ou anormalidades radiológicas cerebrais; nunca ter realizado cirurgias cranianas; ter condições para ser submetido à anestesia geral durante o procedimento, se preciso; não ter evidência de infecção 
local craniana ou cerebral. Para técnicas estereotáxicas guiadas por RM, não pode possuir implantes de objetos metálicos ou de marca-passo cardíaco. O paciente deve ser considerado competente nas esferas legal, mental e psiquiátrica, com envolvimento ativo no processo de decisão de pelo menos um membro próximo da família e um psiquiatra designado para proporcionar atenção primária contínua após a realização do procedimento. Um segundo psiquiatra responsável também deve participar de avaliação independente do paciente para indicação do procedimento. O paciente deve concordar com a realização de uma RM craniana após seis semanas da realização da cirurgia.

Após a verificação do critério de elegibilidade, devem ser realizadas outras avaliações preventivas antes da contemplação do procedimento, incluindo avaliações independentes do paciente e de seus familiares próximos por um especialista em bioética, ou pessoa similarmente treinada, ou por grupo especificamente designado composto por advogados e médicos de diferentes especialidades. O objetivo dessa avaliação é a verificação de que tanto o paciente quanto sua família compreendem plenamente os riscos e benefícios da cirurgia. A avaliação de cada caso, antes e por longo tempo após a operação, por um grupo específico da instituição composto por médicos, advogados e representantes religiosos, também é recomendável como uma segurança final para o paciente. ${ }^{34}$

\section{Técnicas cirúrgicas}

Existem quatro procedimentos psicocirúrgicos geralmente utilizados: cingulotomia, capsulotomia, tractotomia do subcaudado e leucotomia límbica. A capsulotomia anterior é a preferida nos Estados Unidos, enquanto as outras são mais utilizadas na Europa,${ }^{10}$ mas todos os procedimentos envolvem desconexões bilaterais de um ou mais circuitos límbicos, auxiliados pela estereotaxia e por métodos de imagem neurorradiológica, $\mathrm{o}$ que permite alta acurácia na localização das estruturas (mais ou menos $1 \mathrm{~mm}^{3}$ ). Durante a cirurgia, utiliza-se anestesia local, com leve sedação; enquanto os alvos são lesados, os pacientes não referem sensação subjetiva. ${ }^{19}$

\section{Cingulotomia anterior}

Envolve a excisão das fibras anteriores supracalosas do giro do cíngulo, interrompendo o circuito límbico medial..$^{10}$ A cingulotomia implica a termocoagulação estereotáxica, ${ }^{6}$ guiada por RM, do giro do cíngulo anterior e do feixe do cíngulo.

A cirurgia é realizada sob anestesia geral ou local (com sedativo intravenoso) e envolve somente pequena tricotomia. São realizadas trepanações bilaterais de aproximadamente $12 \mathrm{~mm}$ de diâmetro, posteriores 4,5 $\mathrm{cm}$ ao násio e laterais $1,5 \mathrm{~cm}$ (em relação à linha média), feitas juntamente com a utilização de imagens de RM, a fim de uma melhor visualização dos alvos. Originalmente, a localização era feita por ventriculografia aérea (historicamente, o método mais comum para o cálculo de coordenadas na cirurgia estereotáxica) ${ }^{34} \mathrm{e}$ estereotática.

São identificados dois alvos laterais ao giro do cíngulo, entre $2 \mathrm{e} 4 \mathrm{~cm}$ posteriores à borda anterior do corno anterior do ventrículo lateral, e aproximadamente $7 \mathrm{~mm}$ laterais à linha média. ${ }^{10}$ As lesões estendem-se 2 $\mathrm{cm}$ dorsalmente ao corpo caloso e têm entre 8 e $10 \mathrm{~mm}$ de diâmetro lateral, ${ }^{29}$ sendo feitas com a utilização de sondas termocoagulantes por radiofrequência (1-1,5 $\mathrm{mm}$ de diâmetro), ajustadas entre 80 e $85^{\circ} \mathrm{C}$ durante 60 a 100 segundos. A primeira lesão é feita a $8 \mathrm{~mm}$ de profundidade, respeitando o ponto-alvo, e a segunda é feita $10 \mathrm{~mm}$ superficialmente à primeira, resultando em uma área total de aproximadamente 10 por 20 mm. ${ }^{2,10,19}$ Geralmente, após a cirurgia, realiza-se uma TC de crânio para documentação do local da lesão e para descartar a presença de hemorragia..$^{10,19}$ Resultados de exames de imagem com utilização de ${ }^{133} \mathrm{Xe}$ demonstram diminuição do fluxo sanguíneo regional na região frontoparietal em pacientes submetidos à cingulotomia, comparados ao seu estado anterior à cirurgia. ${ }^{10}$

O prognóstico desses pacientes é bem favorável, com indícios de que aproximadamente $25 \%$ deles se encontram funcionalmente bem um mês após o procedimento e outros 31\% apresentam grande melhora em 8,6 anos de seguimento. ${ }^{3}$ Os efeitos colaterais restringem-se a $1 \%$ de casos de epilepsia, controlados com medicação. ${ }^{10}$

Um detalhado estudo de coorte prospectivo, realizado em 1977 no Massachusetts Institute of Technology (MIT), avaliou, mediante testes neuropsicológicos, pacientes submetidos à cingulotomia anterior, demonstrando que, diferentemente das lobotomias frontais anteriormente realizadas, não há evidência de déficits das funções intelectuais ou emocionais nesses pacientes. ${ }^{6}$

No relatório mais recente do Massachusetts General Hospital, descrevendo cingulotomias estereotáxicas realizadas em 34 pacientes com TOC ou distúrbios afetivos maiores desde 1991, consta que 38\% dos pacientes respondem muito bem ao procedimento, $23 \%$ são possivelmente responsivos ao tratamento e $38 \%$ são considerados como não responsivos. A partir desse estudo (e também de outros anteriores com resultados semelhantes), conclui-se que aproximadamente $1 / 4$ dos 
pacientes com TOC intratável por outros métodos podem ser ajudados pela cingulotomia. ${ }^{6,15}$

Outro estudo demonstrou resultado semelhante: 18 pacientes submetidos à cingulotomia foram avaliados após 28,6 meses, demonstrando boa resposta de $28 \%$ ao tratamento e resposta parcial em $17 \%$ dos casos. Após a avaliação de 35 pacientes durante 25 anos, estima-se que $25 \%-30 \%$ dos pacientes se beneficiaram da técnica, apesar de alguns efeitos colaterais como epilepsia (9\%), mania (6\%) e déficit de memória (3\%). ${ }^{10,19}$

A existência de poucos relatos de disfunções cognitivas deve-se às dificuldades em se diagnosticarem mudanças súbitas, como déficit de memória transitório e prejuízo da função cognitiva. ${ }^{15}$ Apesar de não demonstrado com o uso dos testes convencionais, a intervenção neurocirúrgica pode aumentar o número de transtornos do lobo frontal e de funções executivas, isso porque o cíngulo anterior relaciona-se a inúmeras regiões cerebrais, incluindo o circuito fronto-estriato-palido-tálamo-frontal. ${ }^{15}$ Mesmo assim, a cingulotomia mostra-se um bom método de escolha para o tratamento neurocirúrgico de TOC refratário, podendo melhorar de $30 \%$ a $50 \%$ dos sintomas e apresentando poucos efeitos colaterais. ${ }^{19}$

\section{Capsulotomia anterior}

Consiste na lesão da perna anterior da cápsula interna, com a interrupção de conexões entre os lobos orbitofrontais e o núcleo talâmico medial, desligando tanto parte do circuito basolateral límbico como as vias corticoestriatotalâmicas. ${ }^{10}$ Os resultados de estudos anatômicos confirmaram que a perna anterior da cápsula interna contém as radiações talâmicas anteriores (fibras conectando o lobo frontal com o núcleo do tálamo medial e anterior) e o trato corticopontino prefrontal, ${ }^{9}$ envolvidos em sintomas ansiosos.

Estudos pós-operatórios com ${ }^{133} \mathrm{Xe}$ mostraram alteração do fluxo sanguíneo cerebral, ${ }^{10}$ quando comparado aos resultados pré-operatórios. Além disso, um estudo com PET, incluindo cinco pacientes antes e após a cirurgia, demonstrou diminuição da taxa metabólica de glicose no giro orbital e núcleo caudado. ${ }^{26}$ Ambos sugerem o envolvimento de estruturas anatômicas e suas relações na patogênese do TOC.

Como na cingulotomia, a capsulotomia também é auxiliada pela estereotaxia guiada por imagens de RM. As imagens de RM, com secções coronais de $5 \mathrm{~mm}$, geralmente mostram a parte mais anterior dos cornos frontais do ventrículo lateral. Utilizando-se o corte mais anterior, são escolhidos dois alvos em cada hemisfério: o mais inferior é localizado entre 2 e $4 \mathrm{~mm}$ lateral a parte anterior do ventrículo lateral e o superior é $1 \mathrm{~cm}$ acima do alvo inferior. ${ }^{3}$ Há dois tipos de técnica cirúrgica para a realização de capsulotomia: lesão por radiofrequência ou radiocirurgia (gamacapsulotomia).

Na primeira, a cirurgia é feita sob anestesia local e sedação leve, pequenas trepanações bilaterais são feitas logo atrás da sutura coronária, implantando-se eletrodos monopolares com 1,5 mm de diâmetro na área-alvo. São então produzidas lesões térmicas por meio do eletródio durante $75 \mathrm{~s}$, com temperatura próxima a $75^{\circ} \mathrm{C}$. A lesão possui cerca de $4 \mathrm{~mm}$ de largura por 15-18 $\mathrm{mm}$ de comprimento longitudinal. Os pacientes não relatam qualquer desconforto sensorial no decorrer do procedimento. A ocorrência de cefaleia pós-operatória também é incomum, apesar de frequente nos outros procedimentos semelhantes (exceto na cingulotomia). Entretanto, pode ser notado médio comprometimento da iniciativa e do comando mental nos primeiros dois a três meses pós-operatórios, o que parece estar relacionado ao edema perilesional, desaparecendo com a redução deste..$^{10,19}$

A gama capsulotomia (gamma knife) difere quanto à maneira que as lesões são produzidas; nesse tipo de cirurgia elas são feitas por um feixe estreito de irradiação de raios gama provenientes do cobalto com a utilização de estereotaxia, sendo desnecessárias a tricotomia e a craniotomia. O efeito de cada radiação gama individual é desprezível, entretanto o efeito combinado em foco específico produz uma lesão radiocirúrgica. Tal método tem sido utilizado com sucesso por mais de 20 anos no tratamento de outras doenças, com a vantagem de não ser necessária a hospitalização dos pacientes para cuidados pós-operatórios. O risco de indução pela radiação de algum tipo de malignidade não é elevado nesse tipo de procedimento, não existindo relatos desses tipos de casos nas duas últimas décadas. ${ }^{19}$

Há estudos demonstrando a inexistência de grandes diferenças entre esse tipo de cirurgia e a que envolve lesão convencional, ${ }^{10}$ porém é importante salientar que quanto maior o volume irradiado, menor a chance de se predizer como será a lesão, sendo também maior a probabilidade da ocorrência de efeitos adversos. ${ }^{14,19}$

O seguimento de 35 pacientes submetidos a esse procedimento demonstrou melhora em $71 \%$ dos casos. ${ }^{19}$ Em outro estudo, foram comparados resultados pré e pós-operatórios, com seguimento de um ano após a cirurgia: $79 \%$ (19 de 24) obtiveram resultados satisfatórios, concluindo-se também que é improvável a ocorrência de mudanças de personalidade após o procedimento (o que indicaria disfunção do lobo frontal). ${ }^{31}$ Contudo, um trabalho obteve resultados diversos, relatando alterações de personalidade, como perda de iniciativa, diminuição da inibição, humor alterado e conteúdo emocional frívolo. ${ }^{26}$ Uma metanálise 
incluindo oito estudos, com um total de 172 pacientes, concluiu que $67 \%$ foram beneficiados pela cirurgia e apenas $8 \%$ não. ${ }^{27}$

Atualmente, pesquisa-se também o auxílio da eletroestimulação em regiões anteriores da cápsula interna como indutora de efeitos benéficos em pacientes com TOC resistente ao tratamento convencional. O procedimento recentemente demonstrou elicitar alterações da atividade cerebral regional como observado mediante imagens de RM funcional e PET. ${ }^{27,28}$

\section{Tractotomia do subcaudado}

Consiste em lesão da substância inominata em área inferior à cabeça do núcleo caudado, abrangendo tractos de substância branca entre o córtex orbitofrontal e as estruturas subcorticais (tálamo, hipotálamo e amígdalas). O circuito límbico basolateral é interrompido juntamente às vias cortico-estriatais-talâmicas. ${ }^{10,21,29}$

Exames de imagem com ${ }^{133} \mathrm{Xe}$ durante o pós-operatório mostram diminuição do fluxo sanguíneo cerebral nos lobos frontais dos pacientes submetidos à cirurgia em relação aos não submetidos. ${ }^{10}$ Dados de estudo similar, com a utilização de ${ }^{99} \mathrm{Tc}$ hexametil-propilenoamina, demonstram aumento do fluxo sanguíneo cerebral no lobo parietal. ${ }^{10}$ Essas alterações podem estar relacionadas a resultados de que uma diminuição do fluxo sanguíneo cerebral nos lobos do cíngulo e orbitofrontal associa-se a um bom prognóstico e de que um aumento do fluxo sanguíneo estaria, ao contrário, associado a resultados ruins. ${ }^{10}$

O procedimento original consistia em fixação estereotáxica de duas hastes (uma de cada lado) de ${ }^{90} \mathrm{Y}$ radioativo na substância branca ventral, abaixo da cabeça do corpo caloso, no lobo frontal ventromedial (que é a parte que se acredita ser a responsável pelo alívio dos sintomas nesses pacientes), a fim de destruir tecido na região da substância inominata, ${ }^{10}$ utilizando-se para isso a ventriculografia, após a indução de anestesia geral. Assim, provavelmente haveria a destruição de conexões para e do córtex orbital posterior e de estruturas subcorticais, como o giro do cíngulo, amígdala, tálamo e hipotálamo. ${ }^{10}$

Como o metal possui meia-vida aproximada de 60 horas, forma-se uma penumbra conhecida de emissão radioativa de ítrio-90 no tecido cerebral, definindo-se a área cirúrgica lesional, envolvendo a substância inominata. No entanto, alguns estudos posteriores demonstram a frequente não inclusão dessa estrutura no perímetro lesionado em muitos pacientes tratados com sucesso. ${ }^{10}$
Atualmente, as lesões da tractotomia do subcaudado são feitas por meio da utilização de ressonância magnética como guia na introdução estereotáxica da sonda de radiofrequência. $\mathrm{O}$ método atual de colocação da sonda é similar ao da cingulotomia, exceto na realização de três lesões bilaterais, sendo cada uma de aproximadamente $8 \mathrm{~mm}$ de diâmetro, localizadas no mesmo plano coronal, $15 \mathrm{~mm}$ anteriores à base do processo clinoide: a primeira a $6 \mathrm{~mm}$ laterais da linha média e a $10 \mathrm{~mm}$ do assoalho da fossa anterior superiormente, a segunda a 6 $\mathrm{mm}$ laterais da linha média e a $15 \mathrm{~mm}$ do assoalho da fossa anterior superiormente e a terceira e última lesão localizada a $14 \mathrm{~mm}$ laterais da linha média e a $10 \mathrm{~mm}$ superiores ao assoalho da fossa anterior. ${ }^{19}$

Desde 1996, uma nova técnica começou a ser utilizada e vem modificando o procedimento. Esta utiliza o instrumento de Leskell para criar eletrocoagulação por alta frequência com termorregulação. O novo procedimento replica com sucesso o antigo método e não muda as características das lesões, sendo mais barato e produzindo resposta clínica em menor tempo. ${ }^{8}$

$\mathrm{Na}$ Grã-Bretanha, uma análise de tractotomias do subcaudado realizadas entre 1959 e 1995 demonstrou que $34 \%$ dos pacientes com TOC encontram-se em bom estado um ano após a cirurgia. Outros dois estudos revelam melhora em $50 \%$ dos casos..$^{10}$ Também foram feitas análises prospectivas de déficit neuropsicológico pós-cirúrgico, observando-se a existência de certa deterioração em testes sensitivos do lobo frontal e em memória de reconhecimento após a realização do procedimento cirúrgico, sendo ambos prováveis complicações resultantes do edema perilesional, contudo não foi encontrado déficit algum a longo prazo. ${ }^{13}$

\section{Leucotomia límbica}

Trata-se de uma combinação entre cingulotomia bilateral e tractotomia do subcaudado, sendo as menos utilizadas dentre os procedimentos realizados em psicocirurgia. ${ }^{10} \mathrm{~A}$ ideia inicial era de que a dupla lesão poderia produzir melhores resultados funcionais. ${ }^{6} \mathrm{Nas}$ últimas duas décadas, poucos casos têm sido descritos na literatura ${ }^{7}$, entretanto alguns centros consideram-na como primeira escolha no tratamento psicocirúrgico do TOC. ${ }^{10}$

A lesão na região frontal ventromedial visa à interrupção das conexões frontolímbicas (como na tractotomia do subcaudado), enquanto a lesão no cíngulo, à do circuito de Papez. ${ }^{6}$ Para otimização da localização dos alvos a serem lesionados, foi relatado o uso de técnicas de estimulação da substância inominata durante a cirurgia. Uma lesão é feita somente se houver alteração 
da modulação exercida pelo sistema nervoso autônomo (apneia ou pressão arterial) ${ }^{10}$ mesmo assim há controvérsias a respeito da utilidade dessa técnica. ${ }^{10} \mathrm{~A}$ seguir, são feitas trepanações bilaterais $9,5 \mathrm{~cm}$ posteriores ao násio e a $15 \mathrm{~mm}$ da linha média, após tricotomia e anestesia local ou geral.

Ocorre melhora em $89 \%$ dos pacientes com TOC submetidos a essa cirurgia, ${ }^{10}$ além disso, há relatos isolados de eficácia em casos de TOC complicado pela síndrome de Tourette. ${ }^{6,18}$ Alguns seguimentos pós-operatórios demonstram melhora do QI, apesar da possível presença de sintomas transitórios como cefaleia, confusão, letargia e distúrbios esfincterianos. Similarmente às outras cirurgias, o alívio efetivo dos sintomas demora meses para ocorrer. ${ }^{10}$

Em uma avaliação pré e pós-operatória de 26 pacientes, com a tentativa de comparação a um grupo controle com TOC (pareando-se a idade de início da doença, duração e gravidade dela), demonstrou-se que $38 \%$ dos pacientes submetidos à leucotomia apresentam melhora de moderada a intensa quando comparados aos pacientes do grupo controle em tratamento não cirúrgico, num seguimento de seis meses após o procedimento. ${ }^{6}$ Utilizando-se o mesmo grupo controle, os autores também avaliaram características neuropsicológicas em estudo separado, com o achado de que o grupo tratado cirurgicamente não diferia estatisticamente dos controles quanto a funções intelectual e de memória, porém o primeiro teve pior performance numa versão do Wisconsin Card Sort Test, o que é consistente com observações prévias de que lesões frontais possam prejudicar o desempenho nesse tipo de teste. ${ }^{6}$

\section{Conclusão}

A existência de poucos trabalhos comparativos acerca das diferentes técnicas cirúrgicas para o tratamento de TOC limita o conhecimento da repercussão de tais procedimentos na evolução do paciente. Além disso, há diferenças significativas entre os critérios diagnósticos utilizados nos diversos estudos e desinteresse no relato de resultados cirúrgicos negativos, podendo resultar em um viés de aferição e publicação.

Comparando-se as informações de diversos estudos, a leucotomia límbica parece fornecer melhor prognóstico, todavia há evidência considerável de o método de avaliação dos pacientes ser menos criterioso nos grupos que reportaram esses dados. Certamente, nenhuma conclusão definitiva pode ser aferida e necessitam-se de novos estudos.

Para obtenção da eficácia relativa dos diferentes procedimentos, foram comparadas as duas melhores categorias de classificação dos pacientes pós-cirúrgicos (A e B, que representam resposta satisfatória) com as duas piores ( $\mathrm{D}$ e E, aqueles com resultados insatisfatórios). Relata-se uma resposta satisfatória em 8 dos 32 pacientes $(21 \%)$ submetidos à cingulotomia, enquanto 5 (16\%) não apresentam boa resposta; 9 de 18 pacientes (50\%) respondem satisfatoriamente à tractotomia do subcaudado, enquanto 3 (17\%) não demonstram boa resposta. Com a leucotomia límbica, obtêm-se um resultado satisfatório em 30 dos 49 pacientes (61\%), mas 16\% têm uma resposta insatisfatória. Uma revisão recente sobre capsulotomia (Mindus), incluindo oito estudos num total de 172 pacientes com TOC, relatou que 116 (67\%) apresentaram bons resultados após a cirurgia, em relação a 14 (8\%) com resultados insatisfatórios. Mesmo com índices de sucesso comparáveis de capsulotomia e leucotomia límbica, o volume de tecido cerebral lesionado na primeira é menor que na última, o que talvez levasse à preferência na realização daquela na maioria dos centros.

A insuficiência de dados comparando as possíveis terapias para TOC, principalmente quando este se mostra refratário à abordagem convencional, impede a racionalização da escolha do tratamento pelo paciente, que se encontra socialmente excluído por causa de sua condição. Possivelmente, mesmo uma intervenção com riscos de prejuízo neuropsicológico ou de alterações de personalidade seria aceitável considerando-se as circunstâncias. Contudo, as relevâncias éticas de uma indicação psiquiátrica para uma neurocirurgia devem ser revistas, e a realização desta depende não só do paciente, como da disponibilidade da avaliação de uma equipe multiprofissional.

\section{Referências}

1. American Psychiatric Association: Diagnostic and Statistical Manual of Mental Disorders (DSM-IV). 4th ed. Washington: American Psychiatric Press; 1994.

2. Baer L, Rauch SL, Ballantine HT Jr, Martuza R, Cosgrove $\mathrm{R}$, Cassem E, et al. Cingulotomy for intractable obsessivecompulsive disorder. Prospective long-term follow-up of 18 patients. Arch Gen Psychiatry. 1995;52(5):384-92.

3. Ballantine HT Jr, Bouckoms AJ, Thomas EK, Giriunas IE. Treatment of psychiatric illness by stereotactic cingulotomy. Biol Psychiatry. 1987;22(7):807-19.

4. Bebbington PE. Epidemiology of obsessive-compulsive disorder. Br J Psychiatry Suppl. 1998;(35):2-6.

5. Berrios GE. Obsessive-compulsive disorder: its conceptual history in France during the 19th century. Compr Psychiatry. 1989;30(4):283-95.

6. Binder DK, Iskandar BJ. Modern neurosurgery for psychiatric disorders. Neurosurgery. 2000;47(1):9-21; discussion 21-3. 
7. Biver F, Goldman S, François A, De La Porte C, Luxen A, Gribomont B, et al. Changes in metabolism of cerebral glucose after stereotactic leukotomy for refractory obsessive-compulsive disorder: a case report. J Neurol Neurosurg Psychiatry. 1995;58(4):502-5.

8. Carpenter MB. Core text of neuroanatomy. 4th ed. Baltimore: Williams \& Wilkins; 1991.

9. Mercadante MT, Rosário-Campos MC, Shavitt RG, Maia A, Brotto AS, Hounie A, et al. Transtorno obsessivocompulsivo. In: Louzã Neto M, Elkis H, organizadores. Psiquiatria básica. Porto Alegre: Artmed; 2007. p. 315-37.

10. Feldman RP, Alterman RL, Goodrich JT. Contemporary psychosurgery and a look to the future. J Neurosurg. 2001;95(6):944-56.

11. Feldman RP, Goodrich JT. Psychosurgery: a historical overview. Neurosurgery. 2001;48(3):647-57.

12. Hohagen F, Berger M. New perspectives in research and treatment of obsessive-compulsive disorder. Br J Psychiatry Suppl. 1998;(35):1.

13. Kartsounis LD, Poynton A, Bridges PK, Bartlett JR. Neuropsychological correlates of stereotactic subcaudate tractotomy. A prospective study. Brain. 1991;114(Pt 6):2657-73.

14. Kihlström L, Guo WY, Lindquist C, Mindus P. Radiobiology of radiosurgery for refractory anxiety disorders. Neurosurgery. 1995;36(2):294-302.

15. Kim CH, Chang JW, Koo MS, Kim JW, Suh HS, Park IH, et al. Anterior cingulotomy for refractory obsessive-compulsive disorder. Acta Psychiatr Scand. 2003;107(4):283-90.

16. Kitchen N. Neurosurgery for affective disorders at Atkinson Morley's Hospital 1948-1994. Acta Neurochir Suppl. 1995;64:64-8.

17. Korzenev AV, Shoustin VA, Anichkov AD, Polonskiy JZ, Nizkovolos VB, Oblyapin AV. Differential approach to psychosurgery of obsessive disorders. Stereotact Funct Neurosurg. 1997;68(1-4 Pt 1):226-30.

18. Kulisevsky J, Berthier ML, Avila A. Longitudinal evolution of prefrontal leucotomy in Tourette's syndrome. Mov Disord. 1995;10(3):345-8.

19. Jenike MA. Neurosurgical treatment of obsessivecompulsive disorder. Br J Psychiatry Suppl. 1998;(35):79-90.

20. Liu CY, Apuzzo ML. The genesis of neurosurgery and the evolution of the neurosurgical operative environment: part I-prehistory to 2003. Neurosurgery. 2003;52(1):3-19.
21. Malhi GS, Bartlett JR. A new lesion for the psychosurgical operation of stereotactic subcaudate tractotomy (SST). Br J Neurosurg. 1998;12(4):335-9.

22. McDonough M, Kennedy N. Pharmacological management of obsessive-compulsive disorder: a review for clinicians. Harv Rev Psychiatry. 2002;10(3):127-37.

23. Mendlowicz MV, Stein MB. Quality of life in individuals with anxiety disorders. Am J Psychiatry. 2000;157(5):669-82.

24. Micallef $\mathrm{J}$, Blin O. Neurobiology and clinical pharmacology of obsessive-compulsive disorder. Clin Neuropharmacol. 2001;24(4):191-207.

25. Mindus P, Nyman H. Normalization of personality characteristics in patients with incapacitating anxiety disorders after capsulotomy. Acta Psychiatr Scand. 1991;83(4):283-91.

26. Mindus P. Present-day indications for capsulotomy. Acta Neurochir Suppl (Wien). 1993;58:29-33.

27. Nuttin BJ, Gabriëls LA, Cosyns PR, Meyerson BA, Andréewitch S, Sunaert SG, et al. Long-term electrical capsular stimulation in patients with obsessive-compulsive disorder. Neurosurgery. 2003;52(6):1263-72; discussion $1272-4$.

28. Ovsiew F, Frim DM. Neurosurgery for psychiatric disorders. J Neurol Neurosurg Psychiatry. 1997;63(6):701-5.

29. Perse T. Obsessive-compulsive disorder: a treatment review. J Clin Psychiatry. 1988;49(2):48-55.

30. Rück C, Andréewitch S, Flyckt K, Edman G, Nyman H, Meyerson BA, et al. Capsulotomy for refractory anxiety disorders: long-term follow-up of 26 patients. Am J Psychiatry. 2003;160(3):513-21.

31. Salzman L, Thaler FH. Obsessive-compulsive disorders: a review of the literature. Am J Psychiatry. 1981;138(3):286-96.

32. Trivedi MH. Functional neuroanatomy of obsessivecompulsive disorder. J Clin Psychiatry. 1996;57(Suppl 8):26-35.

33. Youmans JR. Neurological surgery. 4th ed. Philadelphia: Saunders; 1994.

\section{Endereço para correspondência}

Leila Maria Da Róz

Rua Arruda Alvim, 136, ap. 61, Pinheiros

05410-020 - São Paulo, SP

Telefone: (11) 7261-9671

E-mail: leilaroz@hotmail.com 